\title{
Le secteur des productions animales en Afrique subsaharienne, des indépendances à 2020 I. Place de l'Afrique subsaharienne dans les échanges mondiaux et évolution du secteur élevage
}

\author{
G. Tacher ${ }^{1}$ L. Letenneur ${ }^{2}$
}

Mots-clés

Production animale - Protéine animale Consommation - Offre - Marché mondial - Afrique au sud du Sahara.

\begin{abstract}
Résumé
Les productions animales de I'Afrique subsaharienne (Ass) contribuent pour une part variable, mais souvent importante, aux économies agricoles des différents pays. Elles représentent en moyenne un tiers du produit intérieur brut agricole (non compris l'élevage). L'Ass contribue pour moins de 2 p. 100 aux échanges mondiaux des produits de l'élevage. Pour les viandes, elle représente 2,2 p. 100 des importations mondiales et participe pour 1,4 p.100 aux exportations. II existe un solde déficitaire de 400 millions de $\$ \cup S$ dont plus de la moitié concerne les viandes blanches. Le déficit dû aux importations est également important pour les produits laitiers, surtout le lait. En revanche, le solde de l'Ass est positif pour les cuirs et peaux. L'analyse de ces flux commerciaux montre qu'ils sont conditionnés par la proximité des partenaires, les contraintes sanitaires et les règlements qui en découlent. La demande régionale en produits animaux est ensuite analysée à partir de la consommation en protéines animales au cours des trente dernières années. Elle montre une faible consommation quotidienne de 9,2 g par habitant, avec une évolution importante des produits consommés, notamment une forte augmentation des viandes de volailles et de porc. Pour les produits laitiers, si la consommation totale augmente de 2 p. 100 par an, la consommation annuelle par habitant diminue $(32,2 \mathrm{~kg}$ en 1994). L'augmentation des productions est souvent inférieure à la croissance de la population humaine ; la production de viandes a doublé en trente ans, mais les importations ont très fortement augmenté. Pour le lait, la production est en forte hausse, mais elle reste insuffisante et nécessite des importations supérieures à 1000 tonnes équivalent lait (teql).
\end{abstract}

\section{INTRODUCTION}

L'élevage contribue pour une part importante aux économies des pays de l'Afrique subsaharienne (Ass) $(1,3,13)$. Au-delà de la fourniture de produits nécessaires à l'homme, il remplit plusieurs fonctions.

C'est une source d'emploi et un moyen de lutte contre la pauvreté. L'essor économique offre aux produits animaux un marché en expansion et de nouvelles possibilités pour l'élevage qui conserve un rôle essentiel en Ass dans le maintien de l'emploi en zone rurale. Il reste indispensable, surtout lorsque l'économie n'est pas assez développée. Dans des conditions écologiques voisines, une famille australienne vit bien là où, sur une même superficie au Sahel, subsistent quatre-vingts familles. Pour soulager les pâtu-

1. Cirad-emvt, Campus international de Baillarguet, 34398 Montpellier Cedex 5, France

2. Cirad-emvt, Campus international de Baillarguet, 34398 Montpellier Cedex 5, France; E-mail : leon.letenneur@cirad.fr rages sahéliens, il faut donner aux trop nombreuses populations qui doivent en vivre une alternative à la migration vers les bidonvilles des métropoles africaines.

L'élevage participe au développement durable de l'agriculture (1, 5, 20), favorise la diversification de ses activités (13), diminue ainsi les risques liés à la production et contribue au maintien des emplois agricoles.

Son rôle bancaire reste indispensable tant qu'un réseau de banques fiables en milieu rural fait défaut.

La diversité considérable des fonctions de l'élevage masque son importance que l'on peut simplement souligner par deux faits :

- le PIB de l'élevage représente en Afrique subsaharienne le tiers du PIB agricole (non compris l'élevage);

- au plan mondial, malgré le faible volume échangé par rapport à la production, les produits animaux représentent plus du double de la valeur des échanges de céréales - qui constituent une sorte de baromètre de référence. 
Enfin, la demande en produits animaux est forte et le restera, même si certains prônent des régimes végétariens, ce qui est à la limite de l'indécence quand cette idée émane de pays où la moyenne de consommation de viande approche du point de saturation (environ $100 \mathrm{~kg} / \mathrm{hab} / \mathrm{an}$ ).

Cette évolution de la demande témoigne du rôle de plus en plus important de l'élevage. La seule fonction de satisfaction de la demande va devenir cruciale pour de nombreux pays.

Après une période de croissance significative de la production au cours des trente dernières années (6) - très inférieure cependant à l'évolution de la consommation - les filières des produits animaux en Afrique subsaharienne ont-elles la capacité de s'adapter, dans le nouveau contexte de concurrence internationale, pour répondre à la forte croissance prévisible des marchés nationaux ou régionaux?

Dans les lignes qui suivront, nous nous attacherons, à la lumière de l'évolution passée, de situer la place de l'Ass dans les marchés mondiaux des produits animaux, de préciser à travers les consommations et les productions les grands traits de l'offre et de la demande des trente dernières années.

Cette première partie sera suivie d'une approche par zonage des régions de production et de consommation. La compétitivité des filières des produits animaux, définie comme la capacité d'acquérir ou de développer des parts de marché, sera analysée en prenant en compte les marchés régionaux.

Enfin, un troisième document rapportera les considérations sur les prospectives pour les deux décennies à venir et les voies possibles pour répondre à la demande croissante en produits animaux. La capacité des filières à répondre au formidable défi du prochain quart de siècle sera examinée dans le contexte régional et international : fournir dans un cadre compétitif et durable, en quantité suffisante, les protéines animales demandées par la population, sans pour autant recourir à un niveau d'importation insupportable pour les économies nationales.

Le présent document a été rédigé à partir d'une étude réalisée à la demande du ministère français des Affaires étrangères : «Etude sur la compétitivité des filières de productions animales en Afrique subsaharienne et à Madagascar » (21).

\section{MATERIEL ET METHODES}

L'étude a porté sur les pays de l'Afrique subsaharienne, incluant l'Afrique du Sud.

A partir de la base de données Faostat (12) et d'annuaires de l'Oaa/Fao $(7,8)$, des éléments ont été sélectionnés et une seconde base de données a été constituée. Elle comprenait les productions et le commerce des animaux et de leurs produits, par pays de l'Ass. Ces données, collectées sur une période de plus de trente ans, ont été regroupées par continents. Les effectifs des populations humaines ont été intégrés pour l'Ass.

Par ailleurs, des enquêtes de consommation et de prix ont été menées dans plusieurs pays. Les données collectées sont en partie analysées dans les chiffres présentés.

Les normes suivantes ont été retenues :

- toutes les valeurs monétaires sont présentées en dollars US ;

- les données statistiques concernant les importations et les exportations, fournies par la Fao et que l'on retrouve tout au long de ce chapitre, sont comptabilisées au prix Fob (Free on board) pour les exportations et au prix Caf (Coût assurance frêt) pour les importations ;

- outre la norme classique de tonne de produit (t), la quantité de viande est exprimée en tonne équivalent carcasse (tec) et celle des produits laitiers en tonne équivalent lait (teql).

\section{- LES ECHANGES MONDIAU X EN VALEU R ET L'AFRIQ U E SU BSAHARIEN NE}

Le commerce en valeur est étudié en premier lieu car il autorise les comparaisons entre les différents produits, ce qui permet de situer les productions animales dans l'ensemble de l'économie.

\section{Le commerce des produits animaux}

Il est constitué par les échanges de toutes les viandes, des produits laitiers, des cuirs et des peaux, des œufs et des laines. Pour des raisons d'homogénéité et de simplification, l'analyse du commerce des produits animaux présentée repose sur 1994, année de référence choisie pour cette étude.

Le commerce de l'ensemble des produits animaux représente 83,3 milliards de \$US soit 1,9 p. 100 du commerce des marchandises, 14,4 p. 100 de l'ensemble des produits de l'agriculture, des pêches et des forêts et 21 p. 100 des produits de l'agriculture. C'est plus du double du commerce mondial des céréales (38,4 milliards). En dollars courants, ce commerce a augmenté de 8,4 p. 100 par an ces trente dernières années.

En 1993, les recettes tirées par les pays en développement de leurs exportations de viandes ont été de 5 milliards de \$US, dont plus de 2 milliards d'exportations d'animaux vivants. En 1994, ils ont participé à 28 p. 100 des exportations mondiales de viande alors qu'ils participaient pour 47 p. 100 à la production. En 1994, les importations mondiales des produits animaux se répartissaient de

Tableau 1

Pourcentage des valeurs des différents produits d'origine animale dans le commerce mondial et dans les importations et exportations de I'Ass (Fao 1995-1996)

\begin{tabular}{lccr} 
& Commerce mondial & Importations Ass & Exportations Ass \\
\hline Toutes viandes & 59 & 68 & 71 \\
Produits laitiers & 28 & 30 & 5 \\
Cuirs et peaux & 5 & 0 & 15 \\
Fufs & 1 & 1 & 1 \\
Laines & 4 & 1 & 8 \\
Autres produits & 2 & - & -
\end{tabular}


la façon suivante : viandes (49,3 milliards), produits laitiers (23,3 milliards), cuirs et peaux (4,2 milliards), laines (3,6 milliards), œufs (1,2 milliard) et autres produits $(1,7$ milliard $)$. Les pourcentages sont rapportés dans le tableau I.

Le solde de ces échanges est positif pour l'Amérique du Nord (2,9 milliards), l'Amérique du Sud (2,2 milliards), l'Europe (7,7 milliards) et l'Océanie (6,9 milliards). Il est négatif pour l'Amérique Centrale (2,6 milliards), l'Asie (12,7 milliards), l'Afrique (2,4 milliards) et l'ex-URSS (21,8 milliards).

Notons que les soldes positifs et négatifs ne correspondent pas en raison des ajustements inévitables et des comptabilisations en prix Fob à l'exportation et en prix Caf à l'importation.

Dans cet ensemble, l'Ass représente 1,9 p. 100 des importations mondiales des produits animaux (soit 1,57 milliard \$US), qui concernent essentiellement les viandes et les produits laitiers. Elles se répartissent selon les pourcentages du tableau I. Les exportations de l'Ass constituent 1,2 p. 100 des exportations mondiales. Si la viande représente plus de 70 p. 100 des exportations, il faut souligner la part importante des cuirs, des peaux et de la laine. Le solde des échanges est déficitaire et s'évalue à - 0,6 milliard en 1994.

\section{Les viandes}

Les échanges de viande constituent 59 p. 100 du commerce des produits animaux. Ils sont répartis de la manière suivante (en \$US) : viande de bœuf (25,8 milliards), viande de porc (12,6 milliards), viande de volaille (6,4 milliards), viande de petits ruminants (3,5 milliards). Le commerce des viandes, dominé par l'Europe, a augmenté de 8,4 p. 100 par an de 1964 à 1994.

Son solde est excédentaire pour l'Amérique du Sud $(+2,1$ milliards), l'Europe (+ 3,3 milliards), l'Océanie (+ 4,4 milliards) et l'Amérique du Nord (+ 2,4 milliards). Il est déficitaire pour l'Amérique Centrale (- 1,1 milliard), l'Asie (- 8,8 milliards) et l'ex-URSS (- 1,7 milliard).

Globalement, le continent africain compte pour 3,1 p. 100 des importations totales de viandes et pour 0,1 p. 100 des exportations. Au nord du Sahara, les importations représentent 0,1 p. 100 des importations mondiales de viandes et les exportations sont considérées comme nulles. Ce commerce reste marginal et déficitaire en Afrique subsaharienne et son solde s'est considérablement détérioré depuis 1974. L'Ass importe 2,2 p. 100 du total des importations mondiales, (1 064 millions \$US), ce qui représente 1,7 p. 100 de ses importations totales. Les exportations n'atteignent que 698 millions, 1,1 p. 100 des exportations totales et 1,4 p. 100 du total des exportations mondiales de viandes.

Les importations de viande bovine et de bovins atteignent à elles seules 586 millions de \$US. Les autres importations se répartissent entre la viande de petits ruminants (225 millions), de volaille (169 millions) et de porc ( 81 millions). Les exportations sont constituées de viande de bœuf (466 millions), de mouton (219 millions), de volaille (4,8 millions) et de porc (3,9 millions).

Ce commerce reste marginal et déficitaire en Ass et son solde s'est considérablement détérioré depuis 1974.

La figure 1 montre la dégradation rapide du solde du commerce des viandes pour toutes les espèces depuis 1974, passant en 20 ans d'un excédent voisin de 300 millions \$US à un déficit proche de 400 millions.

- La viande bovine

Dans le monde, la viande bovine représente plus de la moitié du commerce des viandes. L'Europe constitue 55 p. 100 des exportations totales (2)

Ces exportations totales représentent 31 p. 100 du commerce des produits animaux et 52 p. 100 du commerce des viandes, pour un montant d'exportations Fob de 25,3 milliards \$US. En dollars courants, ce commerce a augmenté de 8 p. 100 par an ces trente dernières années. Il se répartit de la façon suivante : 20 p. 100 pour les bovins vivants, 59 p. 100 pour les viandes fraîches, réfrigérées ou congelées et 21 p. 100 pour les préparations de viande.

Le solde des opérations commerciales (en \$US) sur ces produits est de $+1,3$ milliard en Europe, + 0,5 milliard en Amérique du Nord, + 1,4 milliard en Amérique du Sud, + 3,2 milliards en Océanie, - 0,34 milliard en Amérique Centrale, - 5,1 milliards en Asie et - 0,62 milliard en Afrique (dont - 0,25 milliard pour l'Afrique du Nord et - 0,37 pour l'Ass).

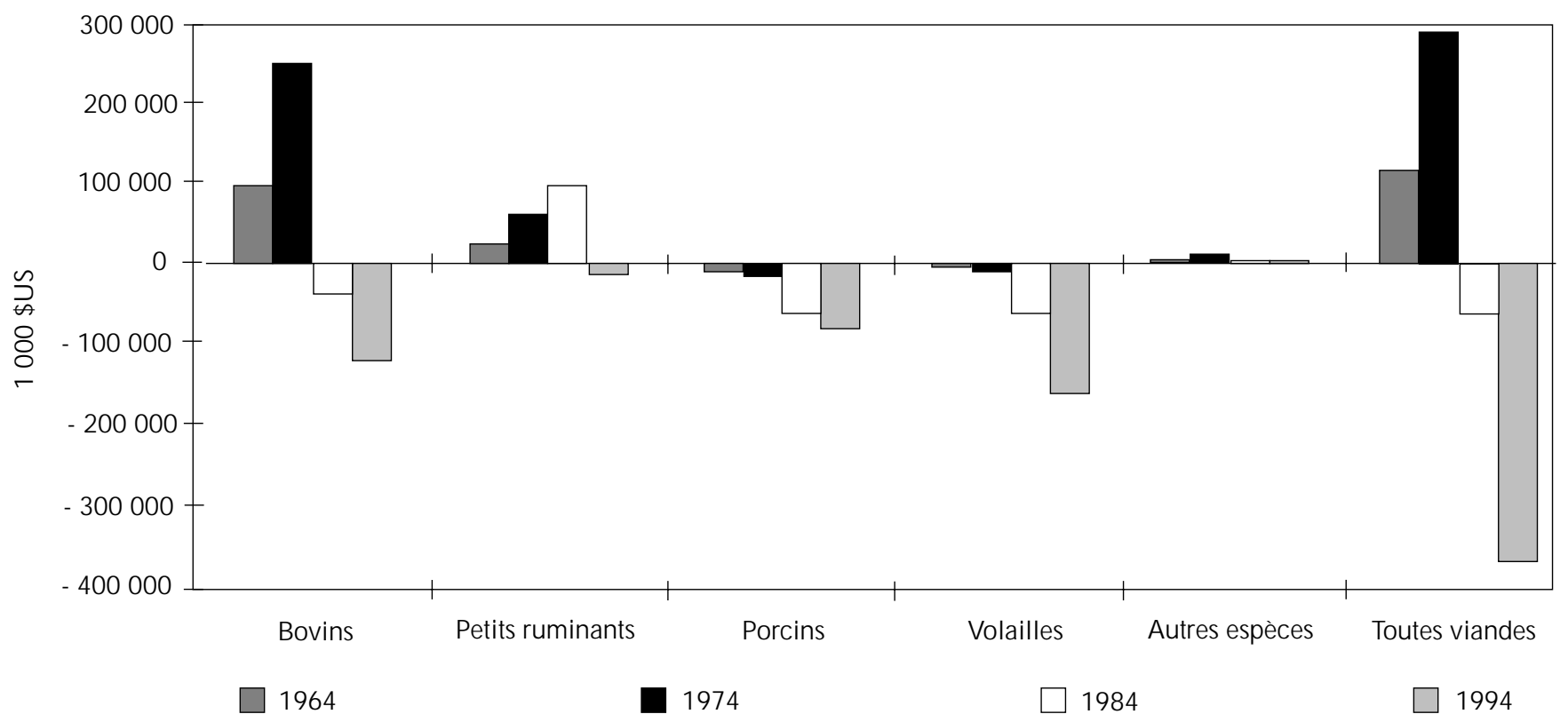

Figure 1 : évolution du solde en viandes de l'Afrique subsaharienne. 
L'Ass montre un déficit en aggravation. Les importations représentent 2,3 p. 100 du commerce mondial des viandes bovines et constituent 55 p. 100 des importations de viandes. Les importations ont ainsi augmenté au rythme annuel moyen de 5,1 p. 100 depuis trente ans. Les exportations sont réalisées pour 56 p. 100 sous forme de bovins vivants, 36 p. 100 de viandes froides, réfrigérées ou congelées, et seulement 8 p. 100 sous forme de conserves et abats. La commercialisation sous forme de viande augmente depuis trente ans.

Le solde montre donc que, exportatrice nette jusque dans les années quatre-vingt, l'Ass est maintenant de plus en plus déficitaire.

- La viande de petits ruminants

Au plan mondial, l'Océanie est le premier exportateur de viande de petits ruminants.

La viande ovine représente 3,7 p. $100 \mathrm{du}$ commerce des produits animaux et 6,3 p. 100 des exportations de viandes pour un montant de 3,1 milliards \$US. En dollars courants, ce commerce a augmenté annuellement de 7,7 p. 100 pour l'exportation et de 7,5 p. 100 pour l'importation ces trente dernières années. La plupart des ovins sont commercialisés sous forme de viandes fraîches, réfrigérées ou congelées (63 p. 100) et 37 p. 100 sont commercialisés en vifs (plus que les bovins, 20 p. 100).

L'Ass occupe une place significative dans le commerce mondial de viande de petits ruminants, avec 6,2 p. 100 des importations. Ses importations constituent 21,1 p. 100 de ses importations totales de viande (la part de l'Afrique du Sud est importante). Elles ont augmenté en dollar courant au rythme annuel de 8,1 p. 100 depuis trente ans. Elles s'effectuent à 37 p. 100 sous forme de viande et à 63 p. 100 d'animaux sur pied. Les exportations représentent 31,9 p. 100 des exportations totales de viande de la zone ( 3 p. 100 seulement sous forme de viande).

\section{- La viande de porc}

$\mathrm{Au}$ plan mondial, l'Europe est en position dominante avec 74 p. 100 des parts du marché. La viande porcine (viandes, préparations et animaux vivants) représente 15,6 p. 100 du commerce mondial des produits animaux et 26,5 p. 100 du commerce des viandes pour un montant de 13 milliards de \$US. Comparée à sa production, c'est la viande la moins échangée (la conservation de la viande n'excède pas six mois). En dollars courants, ce commerce a augmenté de 9,8 p. 100 par an ces trente dernières années.

Le solde des échanges de viande de porc est positif en Europe (+ 2,5 milliards) et en Amérique du Nord (+ 0,6 milliard). Il est déficitaire en Amérique Centrale (- 0,3 milliard) et en Asie (- 1,8 milliards). En Océanie, les exportations sont pratiquement inexistantes. L'Amérique du Sud a un solde négligeable.

En Ass, on observe une augmentation des importations et du déficit. Les importations représentent 0,6 p. 100 du commerce mondial de viande de porc. Elles ont augmenté de 8 p. 100 depuis trente ans pour constituer 7,6 p. 100 des importations de viande. Les exportations représentent 0,6 p. 100 des exportations totales de viandes. Le solde n'a pas cessé de se dégrader depuis trente ans.

- La viande de volaille

L'Europe détient 51 p. 100 des parts du marché mondial, mais le plus grand solde exportateur revient aux États-Unis. Les exportations de viande de volaille représentent 7,6 p. 100 du commerce des produits animaux et 13,5 p. $100 \mathrm{du}$ commerce des viandes pour un montant de 6,4 milliards de \$US. Le poulet constitue 90 p. 100 des viandes de volaille échangées. Le commerce se fait de plus en plus sous forme de découpes ayant plus ou moins de valeur selon les morceaux (les États-Unis, plus gros producteurs, ne consomment généralement que du blanc). En dollars courants, ce commerce a augmenté de 11,8 p. 100 par an ces trente dernières années.

Les soldes de cette activité commerciale sont excédentaires pour l'Amérique du Nord (+ 1,3 milliard), pour l'Europe (+ 0,5 milliard) et l'Amérique du Sud (+ 0,6 milliard). Ils sont déficitaires pour l'Amérique Centrale (- 0,3 milliard) et l'Asie (- 1,1 milliard). Les échanges de viandes de volaille sont pratiquement nuls en Océanie.

En Ass, les importations ont fait un bond important. Elles représentent 2,5 p. 100 du commerce mondial de viande de volaille et comptent pour 16 p. 100 des importations locales de viande. Elles ont augmenté en moyenne de 15,8 p. 100 par an depuis trente ans (10 p. 100 par an depuis 1984). L'Ass achète le quart des exportations européennes, particulièrement des morceaux à bas prix (ailes, pattes, croupions de dinde pour 2 à $3 \mathrm{FF} / \mathrm{kg}$ ). En revanche, sur cette même période, les exportations n'ont pas progressé. La production africaine est destinée à la consommation interne. Le déficit s'est ainsi considérablement creusé depuis trente ans, car la volaille est en train de devenir la première viande consommée dans la région, comme le montre la figure 2.

\section{Le lait et les produits laitiers}

Le commerce d'importation de l'ensemble des produits laitiers représentait en 1994, 23,5 milliards de \$US (10,8 milliards pour le lait et ses dérivés, 3,1 milliards pour le beurre et 9,6 milliards pour les fromages) soit 0,5 p. 100 du commerce des marchandises, 5,9 p. 100 des produits de l'agriculture et 28 p. 100 des produits animaux. En dollars courants, il a augmenté de 9,2 p. 100 par an ces trente dernières années (figure 3 ).

Près de la moitié des échanges mondiaux se fait sous forme de laits et dérivés (47 p. 100), puis viennent les fromages (40 p. 100) et le beurre (13 p. 100).

Le solde des échanges pour l'ensemble des produits laitiers est excédentaire en Europe ( $+4,5$ milliards) et en Océanie (+ 2,3 milliards). Il est déficitaire en Asie (- 3,3 milliards) en Amérique Centrale (- 1 milliard), en Amérique du Sud (- 0,4 milliard), en Amérique du Nord (- 0,1 milliard) et en Afrique (- 1,3 milliard dont 0,4 au sud du Sahara).

En Ass, ce commerce (sur importations) représente 2,0 p. $100 \mathrm{du}$ commerce mondial des produits laitiers et constitue 0,8 p. 100 des importations totales locales. Elles ont été multipliées par 5 depuis trente ans. Le lait en constitue 80 p. 100, les fromages 11 p. 100 (mais 40 p. 100 dans le monde) et le beurre 9 p. 100. L'aide alimentaire, importante pour ce type de produits, explique en partie ces résultats. Les exportations sont pratiquement nulles, elles comptent pour 0,2 p. 100 du commerce mondial et ne représentent quasiment rien par rapport aux exportations de la région. Le solde a évolué au même rythme que les importations et montre un déficit important (figure 4).

\section{Les æufs}

Le commerce des œufs représente 1,2 milliard de \$US soit 1,4 p. 100 du commerce de l'ensemble des produits animaux. En dollars courants, il a augmenté de 5,8 p. 100 par an ces trente dernières années. L'Europe domine les échanges avec 63 p. 100 des importations et 72 p. 100 des exportations mondiales.

Le solde des échanges en œufs est excédentaire pour l'Europe (+ 119 millions) et l'Amérique du Nord (+ 104 millions). Il est négatif pour l'Asie (- 136 millions), l'Amérique Centrale (- 40 millions), l'Océanie (- 1 million) et l'Afrique (- 13 millions). 


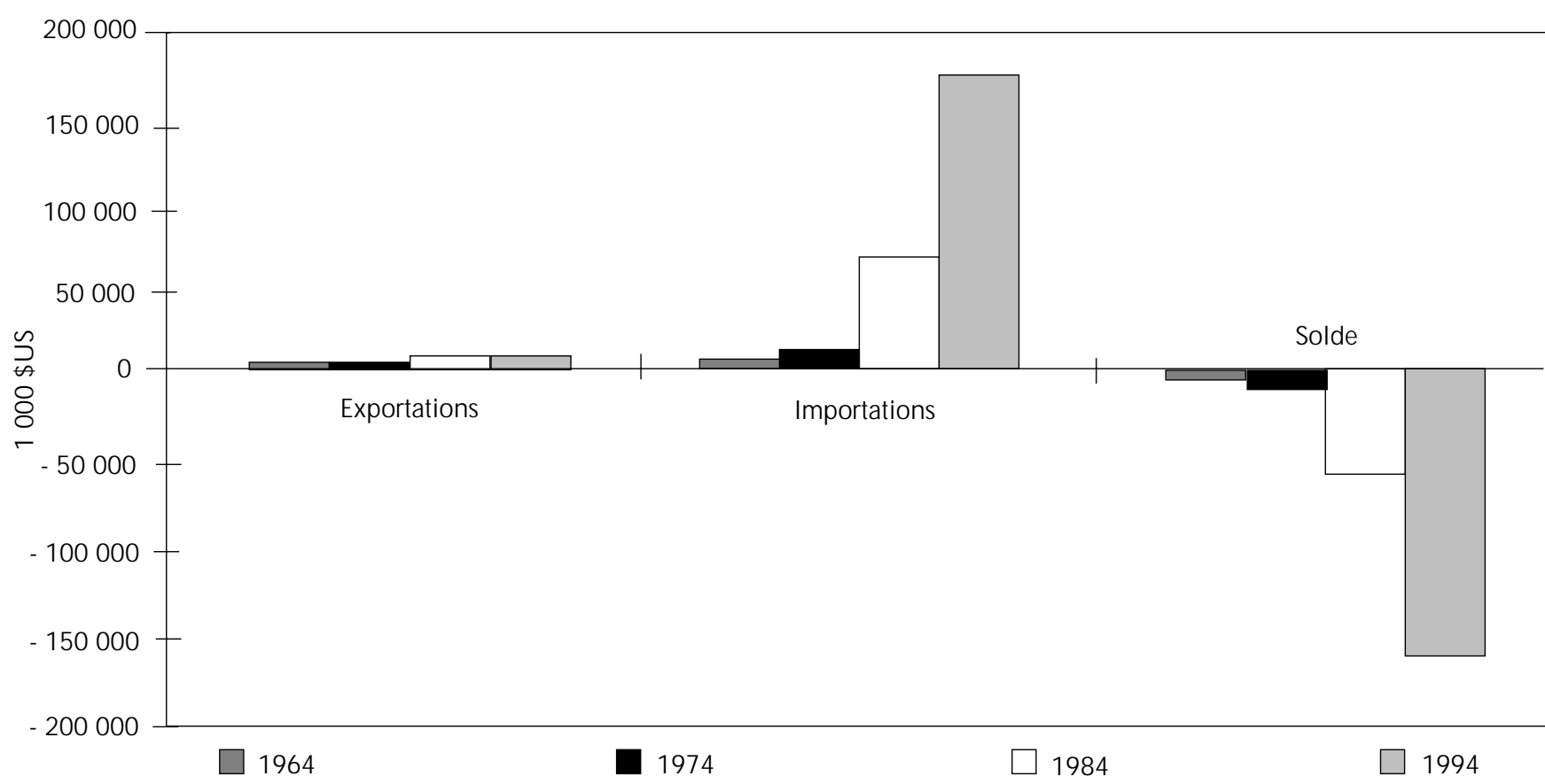

Figure 2 : échanges de viande de volaille en Ass (en \$US).

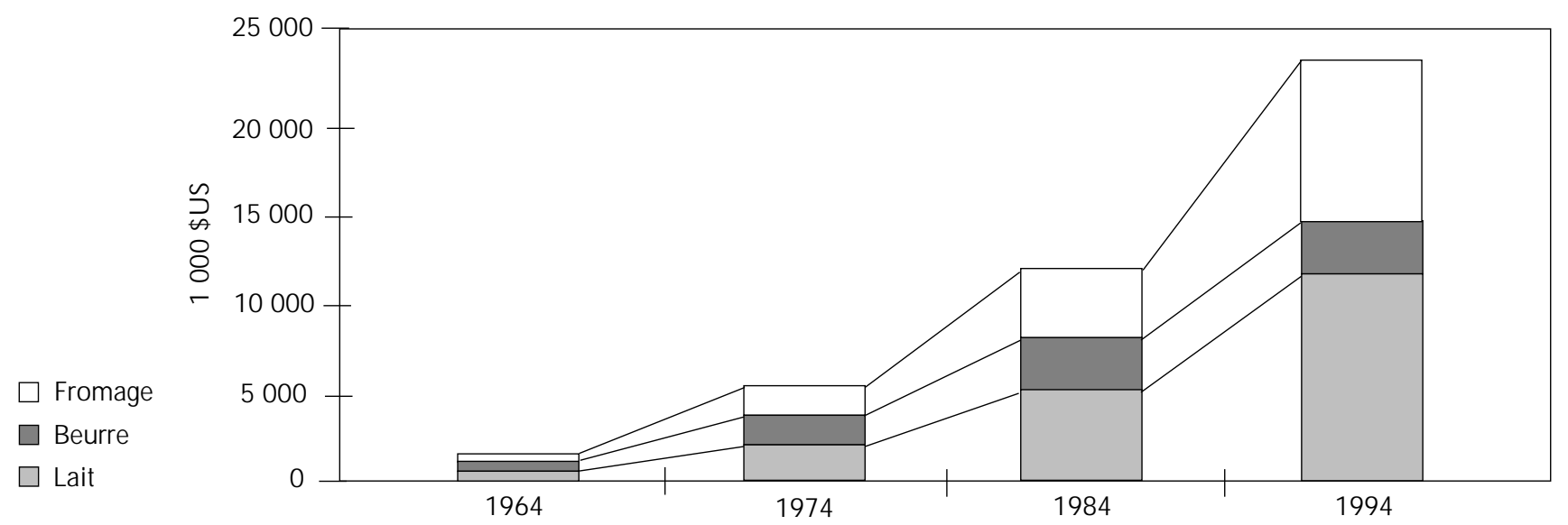

Figure 3 : commerce mondial des produits laitiers (en \$US).

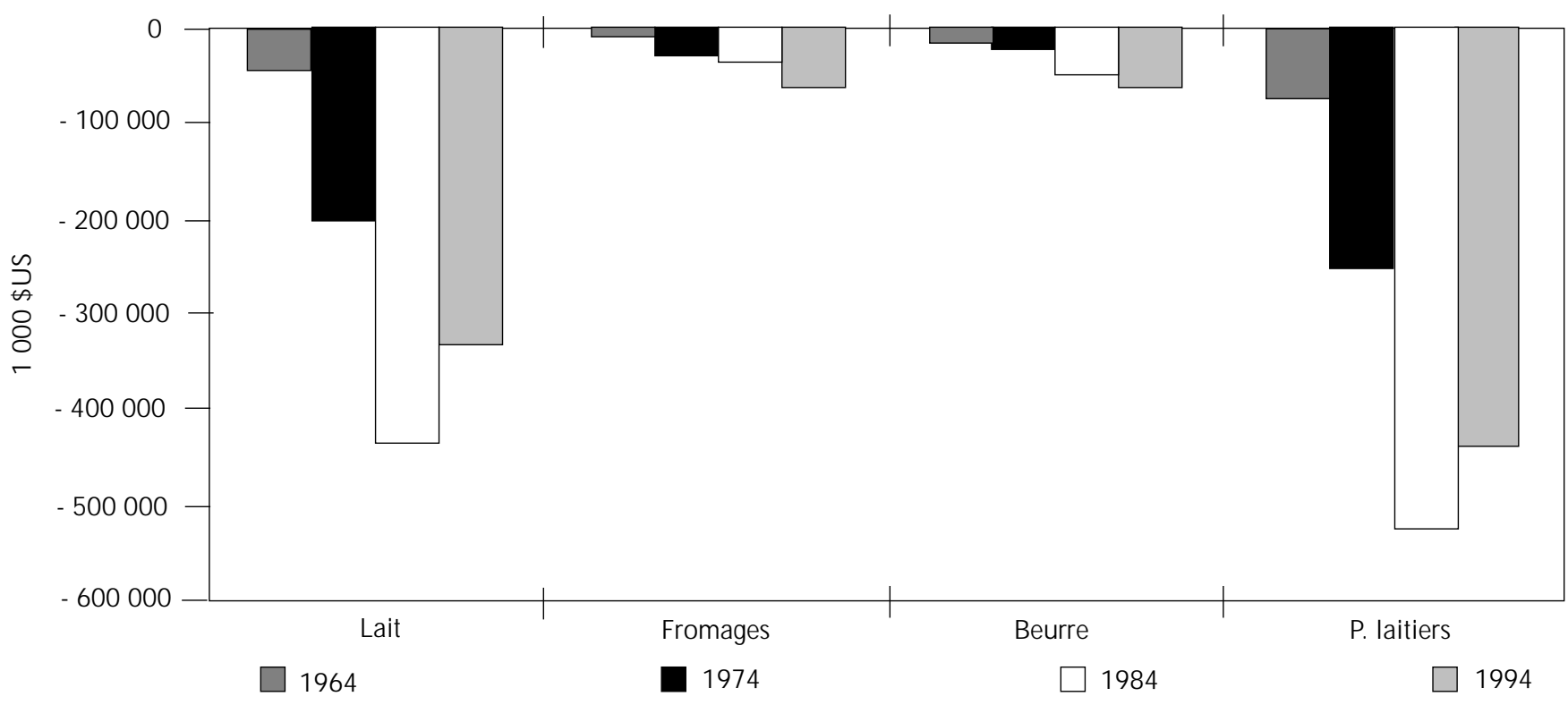

Figure 4 : évolution des soldes des échanges de produits laitiers en Ass (en \$US). 
L'Afrique représente 2,3 p. 100 des importations mondiales d'œufs. L'Ass représente 1,5 p. 100 du commerce mondial avec 12 millions \$US d'importations et 9,5 millions d'exportations. Ce commerce, qui s'effectue surtout sous forme d'œufs en coquille, est principalement interne à la région.

\section{Les laines}

Le commerce mondial des laines représente 3,6 milliards de \$US soit 4 p. 100 du commerce de l'ensemble des produits animaux. L'Océanie est le plus gros exportateur (2,6 milliards). L'Asie est le plus gros importateur (1,8 milliard). L'Afrique représente 1,0 p. 100 des importations et 2,3 p. 100 des exportations mondiales de laines.

En Ass, le commerce est excédentaire de 62 millions de \$US. Les pays importateurs sont l'Afrique du Sud (6,7 millions) et Maurice (11,3 millions). Les pays exportateurs sont l'Afrique du Sud (74 millions), le Lesotho (6 millions) et le Kenya (0,4 million).

\section{Les cuirs et les peaux}

La valeur mondiale des exportations de cuirs et peaux brutes est d'environ 4,2 milliards de \$US soit 5 p. 100 du commerce de l'ensemble des produits animaux.

Les pays en développement des autres continents que l'Afrique se sont dotés d'une importante industrie de transformation, soit en produits semi-finis, soit en articles élaborés (de 1972 à 1992, la valeur des exportations de maroquinerie est passée en Inde de 61 à 1072 millions de \$US).

L'Afrique produit 8 p. 100 des cuirs mondiaux et 14 p. 100 des peaux, mais reste marginale dans le commerce international ( 1,3 p. 100 des cuirs et 6,6 p. 100 des peaux). Elle en exporte pour 155 millions de \$US et en importe pour 77 millions.

L'Ass exporte des cuirs et peaux pour 147,2 millions de \$US et en importe pour 2,6 millions. Les principaux exportateurs sont l'Afrique du Sud (87,7 millions), le Kenya (15 millions), l'Ouganda (13 millions), le Zimbabwe (9 millions), le Nigeria (4 millions), le Burkina Faso, le Sénégal et le Soudan (3 millions).

Les pays développés, pour éviter la pollution provoquée par les tanneries, achètent de plus en plus de produits semi-finis de qualité (wet-blues), difficiles à produire. Cette exigence, jointe au fait que la consommation du cuir augmente, notamment dans les pays anglophones, fait que l'industrie du cuir est en général en difficulté en Afrique.

\section{Les principaux flux des produits animaux sur les marchés Atlantique et Pacifique et les perspectives d'évolution}

L'analyse globale des flux des produits animaux décrits ci-dessus montre le rôle majeur de l'espace dans les échanges commerciaux. C'est la proximité spatiale entre partenaires commerciaux qui explique en grande partie l'importance de leurs relations commerciales. Ainsi en 1992, 41 p. 100 des échanges mondiaux se faisaient à l'intérieur de l'Union européenne (UE) et de l'accord de libre échange Nord américain (Alena). Ces deux ensembles exportent, à l'extérieur de leur zone, seulement 6 p. 100 de la production mondiale de viande $(9,10)$.

Par ailleurs, les flux sont conditionnés par les contraintes sanitaires et les législations des différents pays. L'épidémie de fièvre aphteuse qui sévit encore aujourd'hui a conduit à faire une distinction entre la zone indemne (le marché Pacifique) et la zone contaminée (le marché Atlantique qui comprend l'Union européenne, l'Europe de l'Est, le Moyen Orient et l'Amérique du Sud).
Pour la défense sanitaire des élevages, les stratégies sont différentes dans les deux grandes zones de marchés. Le marché Pacifique adopte un comportement de risque zéro. Il n'importe que des produits «stérilisés » (la chaleur tue le virus aphteux et les viandes en boîte sont admises dans le sens Atlantique Pacifique). L'UE a une politique de risque minimal qui, par la viande désossée, permet des importations de pays où la fièvre aphteuse existe encore. Les nouvelles règles de l'Omc acceptent, définissent et reconnaissent désormais l'état de zone indemne (et non plus de pays indemne). Ce nouveau concept a des conséquences sur les échanges de viandes car de nombreux pays font des efforts considérables pour satisfaire à ces règles internationales.

Le prix de la viande sur le marché Atlantique est en général inférieur de 30 à 50 p. 100 à celui du marché Pacifique, surtout grâce aux subventions de l'UE; cet écart a même atteint 90 p. 100 en 1990. Cette disparité devrait s'effacer avec le temps.

La peste bovine entraîne des difficultés similaires dans les échanges même si elle n'a pas les mêmes répercussions sur le commerce mondial car elle sévit en Afrique et en Asie, deux continents faiblement exportateurs.

L'analyse des différents échanges (21) entre et à l'intérieur des deux zones identifiées ainsi que les grands accords commerciaux régionaux et internationaux (Politique agricole commune de l'Union européenne, Acte final du cycle de l'Uruguay) (16) permettent de tirer les conséquences et les grandes lignes suivantes sur les marchés des produits animaux pour les années à venir $(18,19)$.

- Les accès aux marchés

Ils seront plus faciles et plus importants à long terme.

- Les accords commerciaux internationaux

Ils auront un faible impact sur les prix mondiaux à cause de la faiblesse des échanges par rapport à la production. Les projections des prix sont dues surtout à l'augmentation de la demande, conséquence d'une croissance économique et résultat en partie du cycle de l'Uruguay et de la réforme de la Pac. Cependant, en mettant à part les céréales et le porc, une distorsion apparaîtra entre les prix nationaux et les prix mondiaux.

\section{- La politique commerciale de l'Union européenne}

Elle a accordé des possibilités plus grandes d'accès à son marché pour certaines viandes et pour les produits laitiers. Il faut s'attendre à un accroissement des échanges pour ces produits (principalement le bœuf sur le marché Pacifique). L'augmentation des importations concernera, pour la plus grande part, des contingents tarifaires, dans le cadre d'accords bilatéraux ou préférentiels. L'UE a soutenu l'idée d'un rééquilibrage en faisant des concessions sur les céréales, moyennant un tarif nul pour l'alimentation animale non céréalière (déjà accordée auparavant). Il y a donc eu substitution d'alimentation non céréalière à l'alimentation animale de base en céréales produites au sein de l'UE.

\section{- Les filières}

Elles devront s'adapter. Pour la viande bovine par exemple, 500000 tec (soit le quart des exportations actuelles hors UE) devront chercher un débouché hors subventions à partir de l'année 2000 pour éviter une énorme tension à la baisse sur le marché intérieur.

- Les systèmes de protection classiques des marchés intérieurs

À partir de l'an 2000, les quotas, les monopoles d'importation à un organisme d'Etat, les prélèvements variables sur imports sont destinés à être remplacés par des droits de douane diminuant progressivement. Les différences de prix s'atténueront et les coûts 
des transports feront privilégier les marchés de proximité et les marchés régionaux, d'autant qu'ils seront renforcés par les politiques sanitaires.

- La variabilité des prix

La diversité des échanges et la faible importance des marchés internationaux font qu'il n'existe pas de prix mondial de référence. Les prix varient en fonction des qualités. Volailles et porcs ont des prix calqués sur ceux des céréales, qui varient peu d'un pays à l'autre sauf de façon conjoncturelle. Les viandes de ruminants reflètent plus les coûts de production (selon l'importance des pâturages naturels). La viande est ainsi le régulateur du marché des céréales (la moitié de la viande produite provient d'une transformation des céréales, le quart des céréales produites va à la production de viande) et, plus faiblement, de celui des protéagineux. Sur le long terme, les trois marchés s'autorégulent, tandis que, sur le court terme, les ajustements proviennent du marché mondial. Avec l'Omc, cette autorégulation tendra à se réaliser à l'échelon planétaire.

A côté de cette analyse économique, l'évolution des échanges doit également tenir compte d'autres contraintes.

- Les problèmes de santé animale

En Ass, ils constituent une entrave importante au commerce international $(10,11,14)$. D'une façon générale, on retiendra les maladies de la liste A de l'Office international des épizooties (Oie) présentes en Afrique et plus particulièrement la fièvre aphteuse, la peste bovine, la péripneumonie contagieuse bovine, la peste des petits ruminants et la clavelée chez les ovins et caprins.

Les capacités des services vétérinaires nationaux pour le contrôle des mouvements transfrontaliers des troupeaux représentent également un frein au commerce international. Les pays d'importation vérifient souvent sur place la qualité et la fiabilité des contrôles.

En avril 1994 à Marrakech (Maroc), la réunion des partenaires du Gatt a créé l'Organisation mondiale du commerce (Omc), et a décidé la mise en application de l'accord Sps (sanitaire et phytosanitaire). Dans ce cadre, l'Oie a été retenu comme organisme de référence en matière de santé animale et de zoonoses, ce qui a fait l'objet d'une mise à jour de l'édition du code zoosanitaire (17).

Les récentes réunions de l'Oie ont permis l'adoption de la notion de « zone indemne » et non plus de « pays indemne». Des experts désignés par l'Oie vérifient sur place que les normes très strictes sont respectées pendant un certain temps avant d'autoriser l'exportation. Il y a là un enjeu important pour que l'Ass puisse développer ses échanges.

Pour mieux identifier les effets du commerce mondial et de ces grands accords qui vont conditionner en partie les prix et les choix économiques des voies d'amélioration des productions animales, il est nécessaire de préciser pour l'Ass les évolutions de la demande et de l'offre actuelle.

\section{- L'EVOLUTION DE LA SATISFACTION DE LA DEMANDE EN PROTEINES ANIMALES}

La demande est prise dans son sens le plus large et comprend la demande intérieure (la consommation) et extérieure (les exportations).

\section{La dégradation progressive de la situation alimentaire}

Il existe un écart considérable des niveaux de consommation de protéines animales par habitant entre l'Afrique subsaharienne d'une part, où la situation alimentaire s'est dégradée, le monde et l'Europe d'autre part, où cette consommation progresse respectivement de 24 p. 100 et 42 p. 100 (figure 5).

En Ass, les protéines animales d'origine terrestre consommées par habitant et par jour $(9,2 \mathrm{~g})$ représentent seulement 21 p. 100 des protéines totales (il faudrait y ajouter $3,3 \mathrm{~g}$ de poisson) :

- 5,8 g proviennent de la viande (y compris 1,3 $\mathrm{g}$ de gibier et d'espèces non conventionnelles) (4), soit 2,3 fois moins que dans le monde et 5,3 fois moins qu'en Europe ;

- 2,9 g sont issus de produits laitiers, soit 2,3 fois moins que dans le monde et 6,5 fois moins qu'en Europe ;

\section{- 0,5 g proviennent d'œufs et de produits divers.}

Dans le monde, cette consommation est passée de 20 à 25,8 g. La tendance est sensiblement voisine pour les divers produits animaux : viande, produits laitiers et œufs. En Europe, les protéines animales représentent plus de la moitié (57 p. 100) des apports protéiques. Il est intéressant à noter que l'apport des protéines végétales est remarquablement proche en Ass, en Europe et dans le monde : il est stable sur le long terme, compris entre 41 et $46 \mathrm{~g}$. Une meilleure alimentation nécessite des protéines d'origine animale apportant des acides aminés indispensables, des oligoéléments et des nutriments essentiels.

Aujourd'hui, l'Afrique est globalement malnutrie et sous-alimentée en produits animaux. En fonction des recommandations sur l'état de bonne santé, 200 millions d'Africains environ sont sousalimentés (sans compter les sous-nutritions conjoncturelles lors de catastrophes qui représentent 10 à 15 p. 100 des cas). Le pourcentage de la population sous-alimentée est resté stable (autour de 35 p. 100) entre 1970 et 1990. Dans le même temps, cette proportion a baissé de 19 à 13 p. 100 en Amérique latine et de 40 à 19 p. 100 en Asie.

\section{La consommation de viande}

La figure 6 montre la forte croissance de la consommation de viande par espèce en Ass, mais la figure 7 met en évidence la dégradation de la situation par habitant.

Le rythme de progression des approvisionnements globaux n'a donc pu suivre le taux très élevé de la croissance démographique. L'évolution entre viandes rouges et viandes blanches a été fort différente de 1964 à 1994.

- Les viandes rouges

Depuis 1964 la consommation totale de viande de bœuf a augmenté de 1,7 p. 100 par an (tableau II). En revanche, la consommation annuelle individuelle a diminué de 7,1 à $5,1 \mathrm{~kg}$.

La consommation totale de viande de petits ruminants a augmenté de 2,1 p. 100 par an alors que la consommation annuelle individuelle

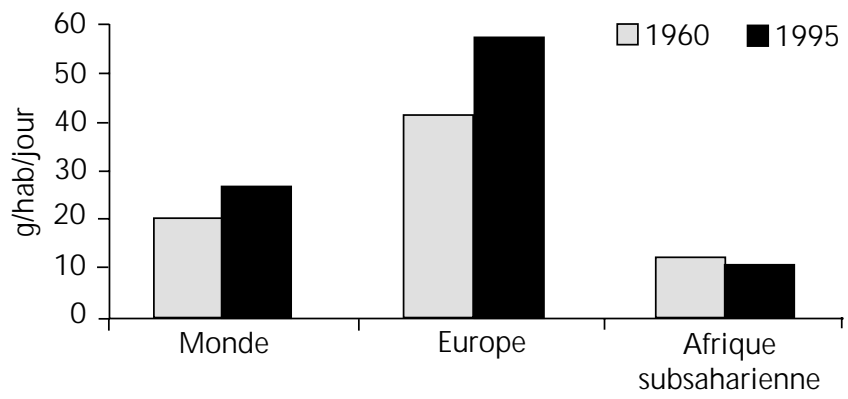

Figure 5 : consommation de protéines animales (en g/hab/jour). 


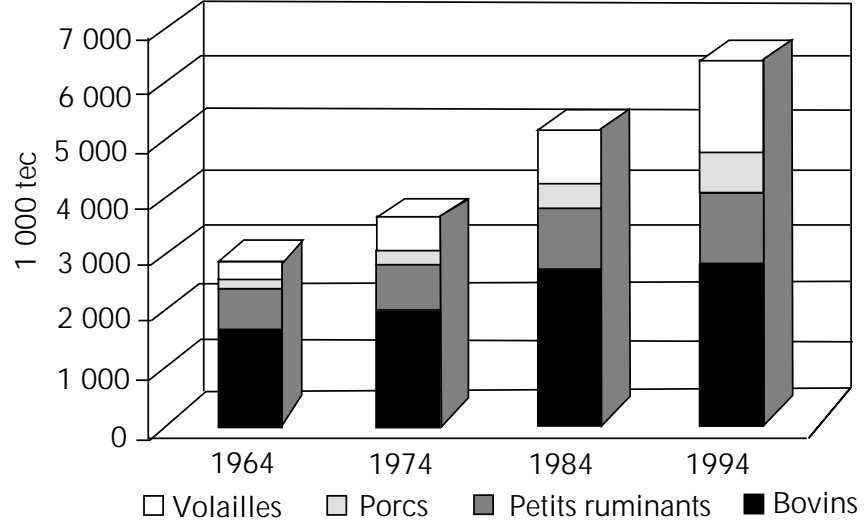

Figure 6 : évolution de la consommation totale de viande en Ass (en tec).

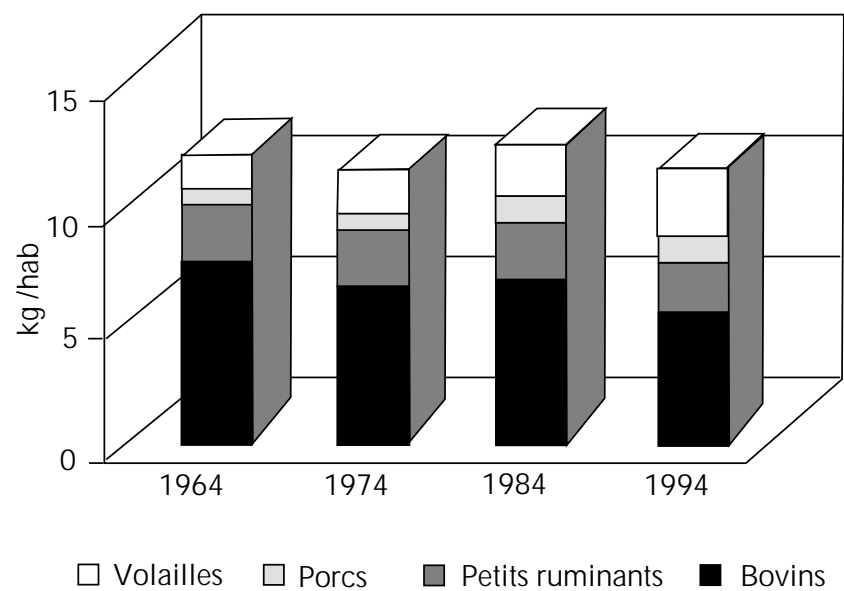

Figure 7 : évolution de la consommation de viande par habitant en Ass (en kg/hab).

est passée de 2,56 à 2,05 kg. La consommation de viande caprine $(1 \mathrm{~kg} / \mathrm{hab} / \mathrm{an})$ est très proche de celle des ovins $(1,06 \mathrm{~kg} / \mathrm{hab} / \mathrm{an})$.

Globalement, depuis trente ans, la consommation totale de viandes rouges a été multipliée par 1,7 mais a baissé de 26 p. 100 par habitant.

- Les viandes blanches

Depuis 1964, la consommation de viande de porc a augmenté de 4,5 p. 100 par an (tableau III) et la consommation annuelle individuelle a augmenté de 0,8 à $1,3 \mathrm{~kg}$.

Pour la viande de volailles, la consommation a augmenté de 5,6 p. 100 par an et la consommation annuelle individuelle est passée de 1,2 à 2,6 kg par habitant.

Globalement, depuis trente ans, la consommation totale de viandes blanches a été multipliée par 4,5 et la consommation par habitant a pratiquement doublé (+ 96 p. 100)

- L'ensemble des viandes

Nous ne prendrons pas en compte la consommation d'autres viandes (camelins, équins, asins, faune sauvage et espèces non conventionnelles) où les imprécisions sont encore plus grandes que pour les espèces conventionnelles. Le lecteur intéressé pourra se reporter à l'étude des auteurs.

Depuis 1964, les viandes blanches se sont substituées progressivement aux viandes rouges. Elles sont ainsi passées de 17 p. 100 à 35 p. 100 de la ration. Mais globalement, la consommation individuelle de viande a baissé de 6 p. 100 (tableau IV).

\section{La consommation des produits laitiers et des oufs}

Depuis 1964 la consommation de produits laitiers a augmenté de 2 p. 100 par an (tableau V) mais la consommation annuelle individuelle a diminué sensiblement, passant de 41,2 à 32,2 kg. Elle se répartit ainsi : 71 p. 100 de lait de vache produit et 6 p. 100 de lait importé, 10 p. 100 de lait de chèvre, 7 p. 100 de lait de brebis et 6 p. 100 de lait d'autres espèces.

La consommation d'œufs a augmenté de 4,3 p. 100 par an et la consommation annuelle individuelle est passée de 1,4 à $2,1 \mathrm{~kg}$ par habitant.

\section{Tableau II}

Consommation totale de viande rouge *

\begin{tabular}{lrrrr} 
& 1964 & 1974 & 1984 & 1994 \\
\hline Bovins & 1788 & 2153 & 2889 & 2943 \\
Petits ruminants & 643 & 693 & 946 & 1184 \\
Total viande rouge & 2431 & 2846 & 3835 & 4127
\end{tabular}

* En 1000 tonnes équivalent carcasse

\section{Tableau III}

Consommation totale de viande blanche *

\begin{tabular}{lrrrr} 
& $\mathbf{1 9 6 4}$ & $\mathbf{1 9 7 4}$ & $\mathbf{1 9 8 4}$ & $\mathbf{1 9 9 4}$ \\
\hline Porcins & 203 & 302 & 406 & 756 \\
Volailles & 291 & 550 & 972 & 1477 \\
Total & 494 & 852 & 1378 & 2233
\end{tabular}

* En 1000 tonnes équivalent carcasse

\section{Tableau IV}

Evolution de la consommation des différents types de viandes (en \% de la consommation de toutes les viandes)

\begin{tabular}{lrr} 
Viandes & $\mathbf{1 9 6 4}$ & $\mathbf{1 9 9 4}$ \\
\hline Bovins & 61,1 & 46,3 \\
Petits ruminants & 22,0 & 18,6 \\
Viandes rouges & 83,1 & 64,9 \\
Porcins & 7,0 & 11,9 \\
Volailles & 10,0 & 23,2 \\
Viandes blanches & 16,9 & 35,1 \\
Total viandes & 100 & 100
\end{tabular}

\section{Tableau V}

Consommation des produits laitiers * et des œufs **

\begin{tabular}{lrrrr} 
& $\mathbf{1 9 6 4}$ & $\mathbf{1 9 7 4}$ & $\mathbf{1 9 8 4}$ & $\mathbf{1 9 9 4}$ \\
\hline Produits laitiers & 10331 & 12459 & 16660 & 18610 \\
Eufs & 340 & 560 & 796 & 1198
\end{tabular}

* En 1000 tonnes équivalent lait

** En 1000 tonnes 


\section{- L'EVOLUTION DE L'OFFRE}

L'offre est prise dans son sens le plus large et comprend la production nationale consommée et les importations.

D'exportatrice, l'Afrique subsaharienne est devenue importatrice. Les succès incontestables en matière de santé animale n'ont pas été suivis par une amélioration suffisante de la productivité pour satisfaire la croissance de la demande.

\section{Les viandes}

\section{Les viandes rouges}

- Les bovins

Entre 1964 et 1994, la population humaine a augmenté de 2.8 p. 100 par an, alors que la croissance numérique annuelle moyenne des effectifs bovins n'a atteint que 1,3 p. 100 (tableau VI). Pour conserver le même niveau de consommation, hors importations, la productivité aurait dû augmenter de 1,5 p. 100 par an.

Les progrès réalisés, limités à 0,3 p. 100 par an, sont liés à l'augmentation des taux d'exploitation permis par un meilleur contrôle de la santé animale. Le poids des carcasses a baissé depuis 1984.

Avec 14,2 p. 100 des effectifs mondiaux, la production de l'Ass ne représente que 5,5 p. 100 de la production mondiale. Malgré la baisse de la consommation individuelle de 1,1 p. 100 par an, l'Ass est passée d'une situation d'exportateur net de 27000 tonnes en 1964 à celle d'importateur net de 20000 tonnes en 1994.

- Les petits ruminants

Avec 18,9 p. 100 des effectifs mondiaux, la production de l'Ass ne représente que 12 p. 100 de la production mondiale.

Les mêmes observations sont à faire (tableau VII) que pour les bovins. La moindre croissance du taux d'exploitation indique que l'effort en matière de santé animale, peu abordé pendant cette période écoulée, doit être intensifié. L'Ass est passée d'une situation d'exportateur net de 23000 tonnes à celle d'importateur net de 8000 tonnes

\section{Les viandes blanches}

Avec 2,4 p. 100 des effectifs mondiaux, la production de viande porcine de l'Ass ne représente que 1,0 p. 100 de la production mondiale. La production de viande de volaille représente 2,5 p. 100 de la production mondiale.

Peu de choses ont été réalisées en matière d'élevage porcin, la croissance importante de la production est surtout due à la croissance des effectifs (tableau VIII).

Pour les volailles, il est difficile de se prononcer au niveau macroéconomique sur les paramètres importants qui ont fait crôtre la

Tableau VI

Effectifs des bovins et tonnage des carcasses produites

\begin{tabular}{lccccc} 
& $\mathbf{1 9 6 4}$ & $\mathbf{1 9 7 4}$ & $\mathbf{1 9 8 4}$ & $\mathbf{1 9 9 4}$ & Tc * \\
\hline Effectifs (millions) & 124,5 & 145,5 & 167,5 & 185,1 & 1,3 \\
Taux d'exploitation (\%) & 10 & 10 & 11 & 12 & 0,6 \\
Poids des carcasses (kg) & 145,8 & 152,3 & 153,3 & 131,2 & $-0,4$ \\
Productivité (kg/animal) & 14,6 & 15,2 & 16,9 & 15,8 & 0,3 \\
Production (1 000 tonnes) & 1815 & 2217 & 2824 & 2914 & 1,6
\end{tabular}

Tableau VII

Effectif des petits ruminants et tonnage de carcasses produites

\begin{tabular}{|c|c|c|c|c|c|}
\hline & 1964 & 1974 & 1984 & 1994 & Tc $*$ \\
\hline Effectifs (millions) & 201,3 & 221 & 274,1 & 321,7 & 1,6 \\
\hline Taux d'exploitation (\%) & 28 & 82 & 77 & 75 & 0,3 \\
\hline Poids des carcasses (kg) & 11,8 & 11,8 & 12,2 & 12,2 & 0,3 \\
\hline Productivité (kg/animal) & 3,3 & 3,3 & 3,5 & 3,7 & 0,3 \\
\hline Production (1 000 tonnes) & 666 & 773 & 968 & 1177 & 1,9 \\
\hline
\end{tabular}

Tableau VIII

Effectif des porcins et production de carcasses de porcins et de volailles

$\begin{array}{lccccr} & \mathbf{1 9 6 4} & \mathbf{1 9 7 4} & \mathbf{1 9 8 4} & \mathbf{1 9 9 4} & \text { Tc * } \\ \text { Production porcine } & & & & & \\ \text { Effectifs (millions) } & 6,2 & 7,9 & 11 & 21,3 & 4,2 \\ \text { Taux d'exploitation (\%) } & 69 & 82 & 77 & 75 & 0,3 \\ \text { Poids des carcasses (kg) } & 47,8 & 46,6 & 47 & 45,9 & -0,1 \\ \text { Productivité (kg/animal) } & 33 & 30,2 & 36,2 & 34,4 & 0,1 \\ \text { Production (1 000 tonnes) } & 203 & 301 & 398 & 733 & 4,4 \\ \text { Production (1 000 tonnes) } & 289 & 543 & 916 & 1331 & 5,2 \\ & & & & & \end{array}$

* Taux de croissance moyen annuel 
production. En effet, on ne dispose pas de statistiques générales sur les parts respectives de l'élevage villageois et de l'élevage modernisé et leurs évolutions. Les efforts importants réalisés en matière de production n'ont toutefois pas permis de suivre la croissance très élevée de la consommation (5,6 p. 100 par an.)

L'Ass est passée pour les porcins d'une situation équilibrée à une situation d'importateur net de 23000 tonnes. Les importations nettes de volailles sont passées de 2000 à 146000 tonnes malgré le développement des productions.

\section{L'ensemble des viandes}

Malgré une augmentation de la production de 3,2 millions de tonnes (elle a plus que doublé en trente ans), la situation générale s'est fortement dégradée puisque d'une situation d'exportateur (48 000 tonnes), l'Ass doit maintenant importer 207000 tonnes de viande (tableau IX).

\section{Les produits laitiers et les oufs}

La production de lait et de produits laitiers est estimée à 17,552 millions de tonnes (tableau X), dont 991000 teql transformées en beurre et fromages et 16,561 millions de tonnes de produits laitiers (lait frais, lait caillé...).

La production de lait en Ass représente 3,1 p. 100 de la production mondiale, celle du beurre 7,8 p. 100, celle des fromages 3,1 p. 100 et celle des œufs 2,7 p. 100 .
Malgré une augmentation de la production de 7,7 millions de tonnes, le volume des importations a été multiplié par 2,2, passant de 477000 tonnes à 1,058 millions de teql (tableau XI).

Pour les œufs, la situation est restée stable et l'Ass est à peu près autosuffisante.

\section{L'amélioration de l'offre}

Ce bilan général de l'évolution de l'offre pourra paraitre pessimiste. Cependant il ne doit pas occulter les efforts entrepris. En trente ans, les productions de viande ont plus que doublé et celles de produits laitiers ont été multipliées par 1,8. Pendant la même période, les villes, qui ont vu leur population multipliée par 4,3 (par 5,2 de 1960 à aujourd'hui), ont pu être ravitaillées et nourries sans problème majeur.

Les expériences acquises pendant cette période permettent de tirer des enseignements qui feront avancer plus rapidement les projets de développement. Leur succès repose sur plusieurs éléments :

- l'approche doit être aussi holistique que possible, avec notamment l'intégration de l'élevage à l'agriculture qui potentialise les productions de chacun; elle ne peut être sectorielle que pour quelques spéculations particulières ;

- la nécessité d'impliquer les professionnels le plus tôt possible (15) ;

- l'intégration de la protection de l'environnement ;

Tableau IX

Evolution des soldes production/consommation des viandes*

\begin{tabular}{|c|c|c|c|c|c|c|}
\hline Viandes & Consommation & $\begin{array}{c}1964 \\
\text { Production }\end{array}$ & Importations & Consommation & $\begin{array}{c}1994 \\
\text { Production }\end{array}$ & Importations \\
\hline Bovins & 1788 & 1815 & -27 & 2944 & 2914 & 30 \\
\hline Petits ruminants & 643 & 666 & -23 & 1185 & 1177 & 8 \\
\hline Viandes rouges & 2431 & 2481 & -50 & 4129 & 4091 & 38 \\
\hline Porcins & 203 & 203 & 0 & 756 & 733 & 23 \\
\hline Volailles & 291 & 289 & 2 & 1477 & 1331 & 146 \\
\hline Viandes blanches & 494 & 492 & 2 & 2233 & 2064 & 169 \\
\hline Total viandes & 2925 & 2973 & -48 & 6362 & 6155 & 207 \\
\hline
\end{tabular}

* En tonnes équivalent carcasse

Tableau $X$

Production de produits laitiers * et d'œufs **

\begin{tabular}{lrrrrrr} 
& 1964 & 1974 & 1984 & 1994 & \multicolumn{1}{c}{ Tc *** } \\
Produits laitiers & 9954 & 11301 & 14367 & 17552 & 1,9 \\
Fufs & 346 & 563 & 794 & 1194 & 4,2
\end{tabular}

* En 1000 tonnes équivalent lait

** En 1000 tonnes

*** Taux de croissance moyen annuel

Tableau XI

Evolution des soldes production/consommation des produits laitiers * et des œufs **

$\begin{array}{lcccccc} & & 1964 & & & & \\ \text { Viandes } & \text { Consommation } & \text { Production } & \text { Importations } & \text { Consommation } & \text { Production } & \text { Importations } \\ \text { Produits laitiers } & 10331 & 9854 & 477 & 18610 & 17552 & 1058 \\ \text { Eufs } & 340 & 326 & 14 & 1198 & 1194 & 4\end{array}$

* En 1000 tonnes équivalent lait

** En 1000 tonnes 
- la recherche systématique de l'optimisation de la productivité du cheptel : les productions animales basées sur le gaspillage de l'espace et la cueillette sont révolues ;

- les mesures d'intensification doivent mieux intégrer les contraintes du milieu ; en recherche, le terrain prime sur les stations.

\section{- CONCLUSION}

L'Afrique subsaharienne comprend une part importante des effectifs animaux. Toutefois, les productions animales font l'objet de peu d'échanges mondiaux. Ce fait tient à une forte consommation intérieure, à une faible productivité du cheptel et aux conditions sanitaires actuelles entraînant des barrières aux exportations.

Ces productions restent cependant insuffisantes pour fournir les apports en protéines d'origine animale nécessaires aux popula- tions de la région. En effet, l'importante augmentation de la démographie a pour conséquence un accroissement considérable de la demande. Celle-ci est mal satisfaite et entraîne une évolution des types de viandes consommés et des modes de consommation.

Avant de proposer des voies d'amélioration utiles à l'augmentation des productions, il faut souligner que l'Ass présente une très grande diversité en ce qui concerne la répartition du cheptel, mais aussi les demandes de produits animaux liées aux concentrations humaines, à leur pouvoir d'achat et à l'accès plus ou moins facile aux produits d'origine animale. Il s'agit d'avoir une analyse géographique cohérente qui puisse amener à proposer des orientations de développement des différents élevages. Pour cela, les pays de l'Ass ont été regroupés par zones complémentaires de production et de consommation. Ce zonage sera présenté dans la deuxième partie de cette étude.

\section{BIBLIO GRAPHIE}

1. WINRO CK INTERNATIO NAL, 1991. Assessment of animal agriculture in sub-saharan Africa. Morrilton, AR, USA, Winrock International Institute for Agricultural Development, $124 \mathrm{p}$.

2. BOUTONNET J.P., SIMIER J.P.,1995. Les viandes. Paris, France, Economica, 110 p. (Coll. Cyclope)

3. CHABEUF N., 1990. Réflexions sur les productions animales en Afrique de I'O uest. In : Réunion préparatoire à la conf. des Ministres de l'Agriculture de l'Afrique de I'O uest, Dakar, Sénégal, 10-14 septembre 1990. S.I., 35 p.

4. CHARDONNET P. ed., 1996. Faune sauvage africaine, la ressource oubliée, 2 vol. Luxembourg, 0 ffice des publications des Communautés européennes, 416 p., $288 p$

5. DE HAAN C., STEINFELD H., BLACKBURN H., 1977. Livestock and the environnement, Finding a balance. Fressingfield, UK, WREN media, $113 \mathrm{p}$.

6. FAO, 1981. Agriculture : horizon 2000. Rome, Italie, Fao, 178 p. (Etudes de développement économique et social, $n^{\circ} 23$ )

7. FAO, 1995. Annuaire du commerce 1994. Rome, Italie, Fao, 385 p. (vol. 48)

8. FAO, 1996. Annuaire de la production 1995. Rome, Italie, Fao, 235 p. (vol. 49)

9. FAO, 1996. Le marché mondial de la viande : situations et perspectives 1995. Rome, Italie, Fao, n. p.

10. FAO, 1996. Rapport de la $16^{\mathrm{e}}$ session du Groupe intergouvernemental sur la viande, Bologne, Italie, 8-10 mai 1996. Rome, Italie, Fao, $13 \mathrm{p}$.

11. FAO, 1996. Obstacles techniques à l'importation de bétail et de viande en provenance de pays en développement et progrès accomplis en ce qui concerne leur réduction en vertu des dispositions de l'U ruguay Round. In : Rapport de la $16^{\mathrm{e}}$ session du Groupe intergouvernemental sur la viande, Bologne, Italie, 8-10 mai 1996. Rome, Italie, Fao, 8 p.
12. FAO/WAICENT. FAOSTAT Statistical databases, http://apps.fao.org. (consulté le 23 septembre 1997)

13. JAHNKE H.E., 1984. Systèmes de production animale et développement de l'élevage en Afrique tropicale. Kiel, Allemagne, Kieler Wissensschaftsverlag Vauk, $279 p$.

14. LACROUTS M., 1969. Problème de la commercialisation du bétail en Afrique. Revue Elev. Méd. vét. Pays trop., 22 : 127-144.

15. MINISTÈRE DE LA COOPÉRATION, 1996. La relance du secteur élevage dans les pays de la zone franc après dévaluation. Paris, France, ministère de la Coopération, 107 p. (Rapport d'étude)

16. OCDE, 1995. Le cycle d'U ruguay : évaluation préliminaire des conséquences de l'accord sur l'agriculture dans les pays de l'Ocde. Paris, France, Ocde, $106 \mathrm{p}$

17. OIE, 1992. Code zoosanitaire international, $6^{\mathrm{e}}$ éd. mise à jour en 1997. Paris, France, O ffice international des épizooties, $450 p$.

18. RO LLAND J.P., 1994. Impact de l'accord du Gatt et de la réforme de la Pac (le cas du marché eurafricain de la viande bovine). Paris, France, Solagral, $182 p$

19. SARN IGUET J., 1990. Effets des importations de viandes du marché mondial sur les échanges de la région Afrique de l'Ouest et centrale 1970-1987. Paris, France, Solagral, n. p. (Réseau stratégies alimentaires, documents du groupe Elevage)

20. STEINFELD H., DE HAAN C., BLACKBURN H., 1977. Livestockenvironnement interactions. Issues and options. Fressingfield, UK, W REN media, $56 \mathrm{p}$

21. TACHER G., LETENNEUR L., 1997. Étude sur la compétitivité des filières de productions animales en Afrique subsaharienne et à Madagascar. Phase 1 : rapport de synthèse. Cirad-Emvt, Montpellier, France/Bdpa-Scetagri, Paris, France, 142 p.

Reçu le 15.9.98, accepté le 21.12.99 


\section{Summary}

Tacher G., Letenneur L. Livestock sector in sub-Saharan Africa, from the Independence's to 2020. I. Sub-Saharan Africa in the world exchanges and livestock sector's trend

Livestock sector represents a variable, but often important, part of the agricultural sector in different countries of sub-Saharan Africa (SSA). It is estimated to about one third of the gross domestic product in the agricultural sector (livestock not included). SSA contributes for less than $2 \%$ of the world exchanges of livestock products. SSA imports $2.2 \%$ of meat and accounts for only $1.4 \%$ of the world exports. The balance of payments deficit amounts to 400 billions US\$ and over the half concerns chicken and pork (white meat). The deficit linked to the milk products is important too. In return, the balance is positive for skin and hides. An analysis of trade flows reveals that they are strongly linked to the proximity of partners, the sanitary constraints and the system of statutes. Then the regional demand of livestock products is analyzed through the animal protein food intakes in the consumption of these last 30 years. This analysis reveals a low daily consumption (9.2 g per capita), with an important evolution of consumed products like chicken and pork. For the milk products, if the total consumption increases of $2 \%$ per year, the annual consumption per capita decreases $(32.2 \mathrm{~kg}$ in 1994). The increase of production is less important than the demographic growth, the livestock production has doubled these last 30 years, but the imports have increased steeply. For milk, the production increases, but not enough, and the imports are superior to 1000 tons eq. milk (teql).

Key words: Animal production - Animal protein Consumption - Supply- W orld markets - Africa south of Sahara.

\section{Resumen}

Tacher G., Letenneur L. El sector de las producciones animales en Africa subsahariana, desde las independencias hasta el 2020. I. Lugar de Africa subsahariana en los intercambios mundiales y la evolución del sector de producción

El sector de las producciones animales en Africa subsahariana (Ass) contribuye en una parte variable, pero a menudo importante, a las economías agrícolas de los diferentes países. Este representa en promedio un tercio del producto interno bruto agrícola. El Ass contribuye en forma limitada en los intercambios mundiales de productos pecuarios. Esta importa 2,2\% de las carnes y participa con $1,4 \%$ de las exportaciones mundiales. Existe pues un saldo deficitario de 400 millones de US\$, de los cuales más de la mitad conciernen carnes blancas. El déficit debido a las importaciones es igualmente importante para los productos lácteos, sobretodo la leche. En revancha, el saldo en Ass es positivo para los cueros y pieles. El análisis de estos flujos comerciales muestra que se encuentran condicionados por la proximidad de los socios participantes, los obstáculos sanitarios y los reglamentos ligados a éstos. Se analiza seguidamente la demanda regional en productos animales, a partir del consumo en proteínas animales durante los últimos treinta años. Esta muestra un consumo cotidiano bajo, de 9,2 g por habitante con una evolución importante de productos consumidos, principalmente un fuerte aumento en las carnes de aves y cerdo. En cuanto a los productos lácteos, si bien el consumo total aumenta de $2 \%$ por año, el consumo anual por habitante disminuye $(32,2 \mathrm{~kg}$ en 1994). La oferta muestra el aumento de la producción, pero este aumento es a menudo inferior al crecimiento de la población humana: el crecimiento numérico anual de los bovinos es de $1,3 \%$, la producción de carnes se ha duplicado en 30 años, pero las importaciones han aumentado fuertemente. La producción de leche se encuentra en fuerte alza, pero permanece insuficiente, requiriendo importaciones superiores a 1000 toneladas equivalentes de leche (teql).

Palabras clave: Producción animal - Proteínas de origen animal - Consumo - O ferta - Mercados mundiales - Africa al sur del Sahara. 